\title{
Optimisation of Customer Demand for Multi-Depot Dynamic Vehicle Routing Problem using Stochastic Demand and Variant of Ant Algorithm
}

\author{
M. R .Nagalakshmi ${ }^{1, *}$ M.Gangadharan ${ }^{2}$, \\ ${ }^{I}$ (Department of Mathematics, Nirmala College, Ranchi University, Ranchi, Jharkhand, India.) \\ ${ }_{2}^{2}$ RDCIS, Steel Authority of India, Ranchi, India)
}

\begin{abstract}
This paper deals with the key issue of management to improve the allocation capability, facilitating the optimization of the objective in minimizing the cost, with the accuracy in dynamic allocation of nearest customers to depot. A method has been proposed to solve the Multi Depot Vehicle Routing Problem (MDVRP) based on Ant Algorithm which is a modified version of the traditional Ant colony Optimization (ACO). Depending on the stochastic demand, distance between depot to customer and customer to customer, the solution to MDVRP is calculated with variant of Ant Algorithm.
\end{abstract}

Keywords: Ant Algorithm, customer demand, Multi Depot Vehicle Routing, Optimization, stochastic demand

\section{Introduction}

Due to technological improvement, Vehicle Routing Problem is giving rise to new problems and as a consequence, this has gained a wide attention among research fraternity. The reasons behind an important component of efficient distribution of goods and transportation system are determination of routing and scheduling a fleet of vehicles. In this competitive challenging world, many industries totally depend on their ability to provide efficient and on-time logistical service. Hence industries or organization need to apply efficient methodologies to effectively schedule a fleet of delivery vehicles with an aim to minimize the total costs involved.

Finding a feasible optimum solution for travelling salesman problem (TSP) shown by [1] as finest instances of vehicle routing problem (VRP) is NP-complete in nature. Set of customers to be visited, customer demands, and time, are few parameters of VRP tend to be stochastic. In literature a different approach to solve vehicle routing problems when the demand is stochastic and design the routes for VRP is studied [2]. The vehicle routing problem with stochastic demand (VRPSD) [3] modeled with capacity and time constraints where customer demands were assumed to be stochastic and other data known in priori. A single depot vehicle routing problem [4] considered a VRPSD where customer demand has been modeled as a stochastic variable independently distributed with known distributions.Multi-Depot Vehicle Routing Problem (MDVRP) is a generalization of the standard Vehicle Routing Problem (VRP), which correlates multiple depots of a company to serve the customers. If the customers are clustered around the depots then the problem can be modeled as a set of various independent VRPs [5]. However, the need to model a multi-depot vehicle routing problem comes into existence if the depots are scattered on the graph along with the customers.

In this paper, the routes are dynamically selected from depot to customer and from customer to customer with a fleet of vehicles based at each depot with an assumption that every customer is served at least once in every cycle. It is constrained by the condition that each vehicle from multiple depots, service the customers assigned to that depot and return to their original depots at the end of their assigned tour while minimizing the total length of all the tours. [6] has attempted an MDVRP taking into account the stochastically processed demand. In the existing routing literature with the MDVRP [7] vehicle routing algorithm is implemented. [8] and [9] deal with Multi Depot Vehicle Routing Problem and generally MDVRP is modeled along with other variants.

Several algorithms are available for VRP. Heuristic algorithms for single and multiple depot vehicle routing problems with pickups and deliveries are tested by [10]. Several stochastic search metaheuristics have been proposed. A few random search techniques include artificial immune algorithm (AIS) [11], genetic algorithm (GA) based approaches [12] and Particle Swam Optimization (PSO) by [13]. [14] introduced ant algorithms that imitate the behavior of real ants. Ant Colony Optimization (ACO) has shown better computational results in existing models [15]. The VRP proposed with minimal fuel consumption as objective is proposed by [16] and studied the functioning of algorithm in an actual road network. A dynamic model on how to optimize the multistage supply chain using the single stage for requirements planning with application to supply chain has been showed by [17]. Cost is an important factor in distribution logistics that directly affects economy of manufacturing industries by minimizing cost productivity of form increased. 
The proposed comprehensive strategy facilitates the optimization of the objective to simplify MDVRP in minimizing the cost, with the accuracy in dynamic allocation of nearest customers to depot using Ant Algorithm. Further to improve the allocation capability, the calculation of travel cost based on distance between depot to customer and customer to customer along with demand a distance matrix is formed and travel routes are fixed to each depot so that the methodology can best be useful in practice. The remainder of the paper is as follows: Section 2 describes in detail the problem description. An overview of solution methodology is provided in section 3, whereas in section 4 results and their discussion are reported. Section 5 summarizes the conclusion.

\section{Problem Description}

In a standard MDVRP each route starts from and ends at the same depot, each customer is served exactly once by a vehicle and the total demand of each route does not exceed the vehicle capacity $v_{h}$. In general if $\mathrm{C}$ is the vertex set, $\mathrm{L}$ is the edge set and cost matrix $\mathrm{D}=\left\{d_{i j}, c_{i} c_{j} \in C\right\}$ corresponding to the distance is defined on $\mathrm{L}$. The vertex set $\mathrm{C}$ is partitioned into two subsets $C_{d}=\left\{c_{1}, \ldots \ldots c_{H}\right\}$ and $C_{c}=\left\{C_{H+1, \ldots \ldots . . .} C_{H+N}\right\}$ respectively the set of Main Depots and the set of customers. Each customer $C_{i} \in C_{c}$ is associated with a non-negative demand $q_{i}$ to be delivered and each depot $C_{h} \in C_{d}$ is associated with a demand $q_{h}=0$. To avoid a vehicle passing two or more depots we assume ${ }^{d_{k l}=\infty}\left(C_{k}, C_{l} \in C_{d}\right)$.

Logistics distribution is dynamic in nature. With increasing number of customers and demand efficient vehicle routing plays a major role for different objective functions like minimizing the cost, travel time [18]. The formulation considered in this paper calculates the dynamic allocation of customers to depot and from one customer to next customer depending on minimum tour length. The problem considered is illustrated in Fig. 1 where central depot (cd) is connected to all main depots and every customer allocated to a depot is interlinked and each customer assigned is connected to that depot.

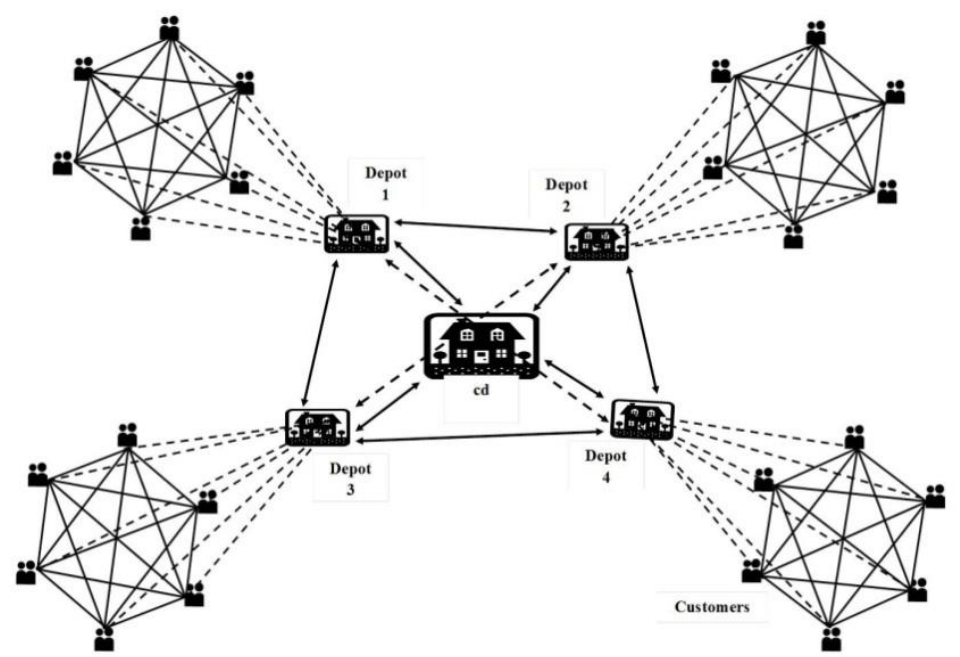

Figure 1 : Interlinking of depots and customers

In order to get a deeper insight of the problem, following assumptions have been made:

1. Every depot is interlinked.

2. Each customer in a depot is interlinked

3. Every customer is served at least once in each service.

4. Each depot has a single vehicle and that the vehicle will never overload its capacity

The variables that are defined for formulating the constraints are as follows:

Tc travel cost

$v_{c} \quad$ vehicle capacity

$M \quad$ all depots

$n_{1}(k) \quad$ number of customers

$n_{2}(k) \quad$ number of depots 
$T c_{i j} \quad$ cost of path $(i, j)$

$c n_{i j} \quad$ additional cost incurred when the customer is not served upon arriving at the location

$c d_{i} \quad$ demand of $i^{\text {th }}$ customer

$d c_{i} \quad$ depot to $i^{\text {th }}$ customer

$t_{n} \quad$ transport cost

$c c_{i} \quad$ customer to customer

$f_{j}^{p r}(r) \quad$ expected cost when preventive restocking is chosen

$d_{j, 0} \quad$ from $i^{\text {th }}$ customer back to depot

$d_{0, j+1} \quad$ again from depot to $j+1^{\text {th }}$ customer

The objective function is computed as follows:

$$
\begin{gathered}
T c=\min \left[\sum_{m=1}^{M} \sum_{i=0}^{n_{1}(k) n_{2}(k)} \sum_{j=0} a_{i j} d_{i j}\right] T c_{i j}+c n_{i j} \\
{[A]=\text { if }\left\lfloor a_{i j}\right\rfloor=0 \quad \text { no arc between } i \text { and } j} \\
=1 \quad \text { If arc is between } i \text { and } j
\end{gathered}
$$

As detailed by [19] in case of preventive restocking when the vehicle do not have sufficient load to serve next customer, it performs back and forth trip to the depot for completing the delivery at the customer .

$$
\begin{array}{lc}
f_{j}^{p r}(r)=d_{j, o}+d_{0, j+1}+\sum_{m=1}^{M} \sum_{D=1}^{D_{n}} f_{j+1}\left(v_{c}-D\right) p_{j+1, D} \\
\sum_{j=1}^{v} x_{0, j, v_{h}}=1 & \forall v_{h} \in\{0,1,2, \ldots n v\} \\
\sum_{j=1}^{v} x_{j, 0, v_{h}}=1 & \forall v_{h} \in\{0,1,2, \ldots . n v\} \\
\sum_{j=1}^{v} \sum_{k}^{n v} x_{i, j, k}=1 & \forall i \in v \text { and } i \neq 0 \\
\sum_{j=1}^{v} \sum_{k}^{n v} x_{j, i, k}=1 & \forall i \in v \text { and } i \neq 0
\end{array}
$$

Equations (3) and (4) represent that the vehicle visits only the customers from the depot and back to depot. Equations (5) and (6) infers that every customer is served only once in each service.

$$
\operatorname{MinT} c_{\text {cust }}=\sum_{i=0}^{n} \sum_{j=0}^{m} T c_{i j}
$$

Condition-1

If initial tour starts from depot

Condition-2

Reloading

$$
\operatorname{MinTC}_{c_{i} c_{j}}=\sum_{i=0}^{n} \sum_{j=0}^{m} T C_{c_{i} c_{j}}
$$




$$
\begin{aligned}
T_{c_{i}} & =\sum_{i=0}^{m} D_{c_{i}} \text { Dist } \times C D_{i} \times T_{n} \\
T_{c_{i}} & =\sum_{i=0}^{m} C C_{i} \text { Dist } \times C D_{i} \times T_{n}
\end{aligned}
$$

Equations (7) and (8) provide the minimum cost from depot to customer and minimum cost from customer to customer. Equations (9) and (10) states the travel cost based on distance from depot to customer and from customer to customer. Combining the distance, travel cost and the demand, customers are assigned considering the conditions that the initial tour starts from depot and also the condition of the threshold value, in case the vehicle is to be reloaded.

\section{Solution Methodology}

Metaheuristic search technique can be effectively applied to complex optimization problems with a slight modification. Our approach adopts Ant Colony Optimization technique to get good quality solution. The algorithm involves nature inspired technique. From the behaviour of artificial agents called ants which independently find the shortest path from a food source to their nest [20]. With some initialization criteria, the ants randomly move from starting nodes over the feasible nodes [21]. Now by state transition rule [22], visit to next city is followed and the ants are allowed to move to unvisited cities. Several improved ant colony optimization algorithm for resolving complexities in the search techniques have been proposed [23][24]. A dynamic vehicle routing problem is analyzed and strategy based on Ant colony System is proposed by [25] on a set of benchmarks available. To incorporate the strategy in the existing ant algorithm, a technique has been embedded in this research. To illustrate the working, a detailed flowchart has been illustrated in Fig. 2.This methodology determines minimum cost delivery routes depending on minimum tour length from customer to customer and depot to customer. Sometimes the vehicle returns to the depot if constraints would be violated which is termed as preventive restocking. In this preventive restocking when the vehicle do not have sufficient load to serve next customer, it performs back and forth trip to the depot for completing the delivery at the customer. Depending on the customer demand using stochastic probability, distance between depot to customer and customer to customer, the solution to MDVRP is calculated using stochastic demand and the sequence of the customers is followed using variant of Ant Algorithm.

First, the minimum cost from depot to customer is considered and the demand is fulfilled. The remaining load is served to next nearest customer though the travel cost is more i.e., only comparing with the nearest customer. In the process when the remaining load is 0 the vehicle goes back to depot for reloading. Checking with the threshold value of the vehicle and other mentioned methodology the customer demand is fulfilled.
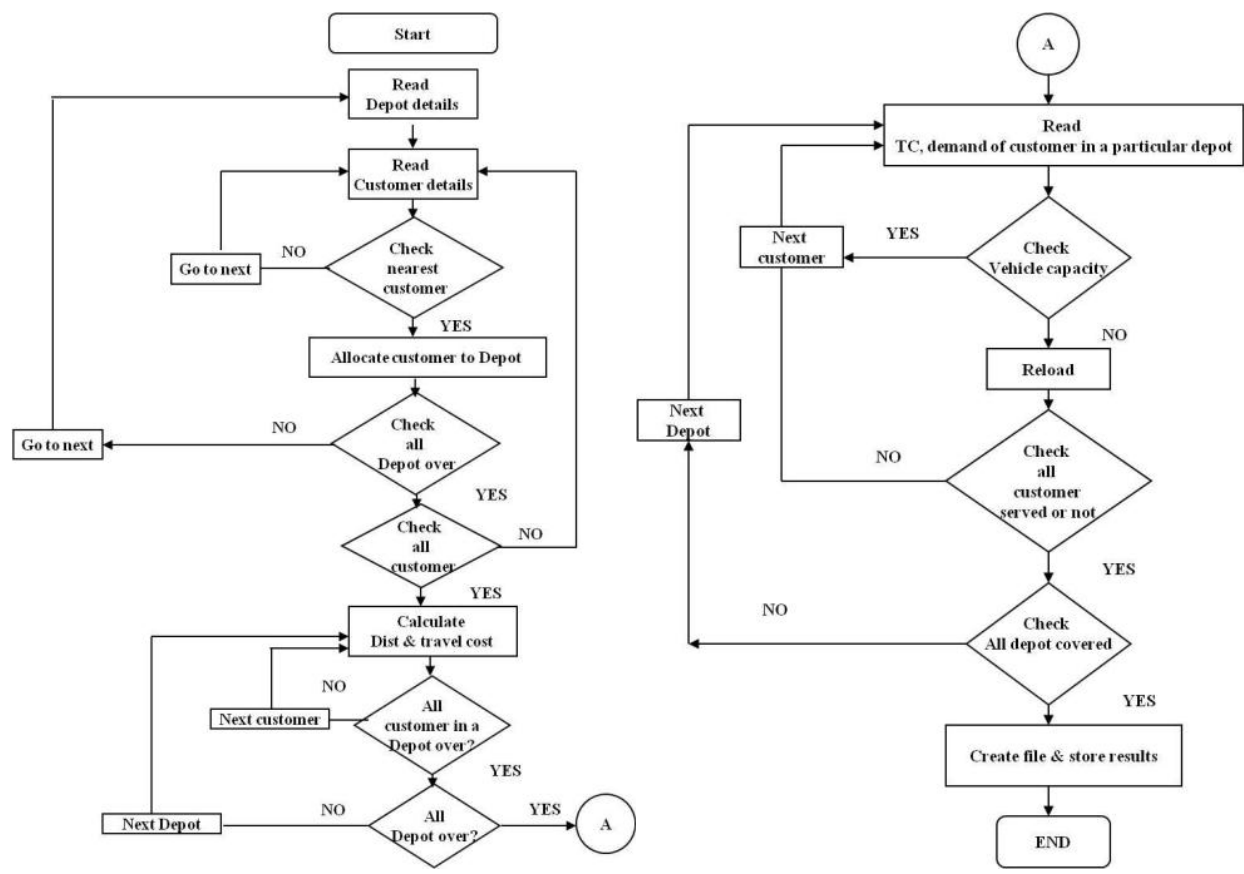

Figure 2: Model flow diagram 


\section{Computational Results and Discussion}

This section exemplifies the problem considered with a view to demonstrate the efficacy of the proposed technique. Based on the strategy developed, a comprehensive distribution and logistics plan is aimed at minimizing the cost associated with the vehicle tours while achieving maximum annual profit. Table 1 shows the customers allotted to Depot 1 along with their demand. In general, first the customer demographical area is measured from the location of the allocated depot as described in Table 1. Based on the distance and demand, travel cost is calculated for customers of Depot 4 and has been illustrated in Table 2.

Table-1: Demographical survey of customers and demand

\begin{tabular}{|l|l|l|l|l|}
\hline Sl.No & Customer & X axis & Y axis & Demand \\
\hline 0 & 17 & 4.8 & 12.3 & 10 \\
\hline 1 & 19 & 5.3 & 11.4 & 12 \\
\hline 2 & 22 & 5.6 & 11.1 & 6 \\
\hline 3 & 24 & 5.8 & 12.8 & 8 \\
\hline 4 & 31 & 6.5 & 13.6 & 15 \\
\hline 5 & 33 & 6.6 & 12.5 & 17 \\
\hline 6 & 37 & 7.2 & 11.1 & 9 \\
\hline 7 & 46 & 8.2 & 11.4 & 18 \\
\hline 8 & 59 & 6.1 & 10.6 & 9 \\
\hline 9 & 60 & 6.6 & 11.2 & 8 \\
\hline 10 & 61 & 6.3 & 10.8 & 7 \\
\hline 11 & 66 & 8.5 & 10.8 & 8 \\
\hline 12 & 67 & 8.1 & 13.1 & 9 \\
\hline 13 & 68 & 8.1 & 12.7 & 10 \\
\hline
\end{tabular}

Table-2: Travel cost of customers

\begin{tabular}{|l|l|l|l|l|l|}
\hline Sl.No & Depot to & Customer & Distance & Travel Cost & Demand \\
\hline 0 & 4 & 6 & 2.06 & 319.16 & 10 \\
\hline 1 & 4 & 7 & 1.77 & 137.33 & 5 \\
\hline 2 & 4 & 8 & 1.77 & 164.8 & 6 \\
\hline 3 & 4 & 9 & 0.57 & 61.38 & 7 \\
\hline 4 & 4 & 12 & 0.57 & 87.68 & 10 \\
\hline 5 & 4 & 13 & 1.24 & 230.07 & 12 \\
\hline 6 & 4 & 15 & 0.10 & 12.4 & 8 \\
\hline 7 & 4 & 16 & 1.46 & 203.12 & 9 \\
\hline 8 & 4 & 27 & 2.48 & 462.01 & 12 \\
\hline
\end{tabular}

Table 3 demonstrates the sequence of the customers followed using Ant Algorithm. This information determines minimum cost delivery routes depending on optimum delivery as per customer demand and minimum travel cost. The vehicle returns to the depot while considering capacity constraints before completing the delivery to the next nearest customer. Depending on the customer demand using stochastic probability, distance between depots to customer and customer to customer is calculated using stochastic demand. Now using variant of Ant Algorithm, the order of the customers is followed. First, the minimum cost from depot to customer is considered and the demand is fulfilled. The remaining load is served only to next nearest customer though the travel cost is more. In this process when the remaining load is 0 , the vehicle goes back to depot for reloading and again the methodology is followed to fulfill the customer demand.

Table-3: Sequence of customers followed using proposed strategy

\begin{tabular}{|l|l|l|l|l|l|l|}
\hline SI.No & Travel from & Travel to & Distance & Travel Cost & Demand & No of visit \\
\hline 0 & 4 & 15 & 0.10 & 12.40 & 8 & 1 \\
\hline 1 & 15 & 9 & 0.14 & 15.34 & 7 & 1 \\
\hline 2 & 9 & 7 & 0.71 & 54.80 & 5 & 1 \\
\hline 3 & 4 & 12 & 0.57 & 87.68 & 10 & 1 \\
\hline 4 & 12 & 8 & 0.14 & 13.15 & 6 & 1 \\
\hline 5 & 4 & 16 & 1.46 & 203.12 & 9 & 1 \\
\hline 6 & 16 & 6 & 1.98 & 306.88 & 10 & 1 \\
\hline 7 & 4 & 6 & 1.98 & 230.07 & 12 & 1 \\
\hline 8 & 4 & 27 & 2.48 & 462.01 & 12 & 1 \\
\hline
\end{tabular}




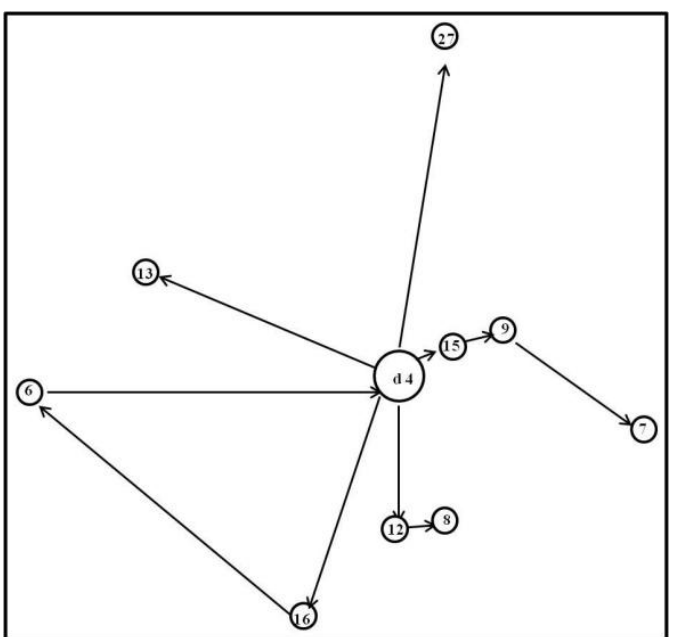

Figure 3: The route followed for minimum travel cost is illustrated for depot $4\left(d_{4}\right)$ and customers allocated.

The algorithm was tested using visual $\mathrm{C}++$, version 2010 . The algorithm was run on a randomly generated data with 68 customers and 5 depots with one central depot and the distances of customers and depots were calculated from demographical map of Ranchi while considering the homogeneous vehicle each with capacity of 20 tonnes.

\section{Conclusion}

This research considers the stochastic demand for multi-depot dynamic vehicle routing and aims at minimizing the cost associated with the vehicle tours. An innovative approach using variant of ant algorithm facilitates the optimization of the objective with the accuracy in dynamic allocation of nearest customers to depot, and finally serving the customers using the solution methodology based on travel cost and distance from customer to customer and depot to customer. Since the strategy here is a new problem, we hope our results and the algorithm developed will enable other researchers to produce comparative results.

\section{References}

[1]. Tan, K.C., Lee, L.H., and Ou, K., : Artificial intelligence heuristics in solving vehicle routing problems with time window constraints. Engineering Applications of Artificial Intelligence, vol. 14, 2001, 825-837

[2]. Dimitris J.Bertsimas.,: A vehicle routing problem with stochastic demand. Operations Research Society of America. vol 40, 1992, 3

[3]. Cheong, C.Y., Tan, K.C., Liu, D.K., Xu, J.X., : A multi objective evolutionary algorithm for solving vehicle routing problem with stochastic demand. IEEE congress on Evolutionary Computation., 2006, 1370-1377

[4]. Nagalakshmi, M.R., Mukul Tripati., Nagesh Shukla., Tiwari, M.K., : Vehicle routing problem with stochastic demand (VRPSD): optimization by neighbourhood search embedded adaptive ant algorithm (ns-AAA). Int. J. Computer Aided Engineering and Technology, vol-1, No.3, 2009, 300- 321

[5]. Hjorring, C., : The Vehicle Routing Problem and Local Search Metaheuristics. Chapter 2. PhD thesis. Department of Engineering Science, The University of Auckland. 1995

[6]. Chan Yupo, Carter William, B., Burnes, Michael, D., : A multiple-depot, multiple-vehicle, location-routing problem with stochastically processed demands. Computers and Operations Research 28 ,2001, 803-826

[7]. Golden, B., Magnanti, T. L., Nguyen, H. Q., : Implementing Vehicle Routing Algorithms. Networks. 7, 1977, 113-148

[8]. Benoit Crevier, Jean-Francois Cordeau, Gilbert Laporte., : The multi -depot vehicle routing problem with inter depot routes. European Journal of operational Research 176, 2007, 756-773

[9]. Byu, Z-Z Yang., J-X Xie., : A parallel improved ant colony optimization for multi-depot vehicle routing problem. Journal of the operational Research Society. 62, 2011, 183-188

[10]. Gabor Nagy, Said Salhi.,: Heuristic algorithms for single and multiple depot vehicle routing problems with pickups and deliveries. European Journal of Operational Research. 162(1), 2005, 126- 141

[11]. De Castro, L.N., and Timmis, J.I., : Artificial Immune Systems: A New Computational Intelligence Approach. September, Springer-Verlag, London, 357,2002

[12]. Baker, B.M., Ayechew, M.A : A genetic algorithm for the vehicle routing problem. Computers and Operations Research 30, 2003, $787-800$

[13]. Bonabeau, E., Dorigo, M., and Theraulaz, G., : Swarm Intelligence from Natural to Artificial systems. A volume in the Santa Fe Institute studies in the science of complexity. Oxford University press , 1999

[14]. Dorigo, M.,:Optimization, Learning and Natural Algorithms. Ph.D. Thesis, Dipatimento di Elettronica, Politecnico di Milano, IT. 1992.

[15]. Reimann, M., : Analyzing a vehicle routing problem with stochastic demands using ant colony optimization at advanced OR and AI methods in transportation. Poznan. 2005, 764-769

[16]. Zhifeng Lang., Enjian Yao., Weisong Hu., Zheng pan.,: A vehicle routing problem solution considering alternative stop points. Elsiver.Procedia-Social and Behavioral sciences 138, 2014, 584-591

[17]. Stephen C.G., David B.k., William,B.H.,: A dynamic model for requirements planning with application to supply chain optimization. Operations Research. vol.46, 3, 1998, 35-49

[18]. Psaraftis, H.N.: Dynamic vehicle routing: Status and prospects. Annals of Operations Research 61, 1995, 143-164 
[19]. Yang, W., Mathur, K. and Ballou, R.H. Stochastic vehicle routing problem with restocking, Transportation Science, Vol .34, No 1, 2000, 99-112

[20]. Beckers, R., Deneubourg, J.L ., Goss, S : Trails and U-turns in the selection of the shortest path by the ant Lasius Niger. J. Theoretical Biology. Vol. 159, 1992, 397-415

[21]. Agrawal, S., Tiwari, M.K : A collaborative ant colony algorithm to stochastic mixed-model U- shaped disassembly line balancing and sequence problem. International Journal of Production Research, available online at DOI: 10.1080/00207540600943985,2006

[22]. Dorigo, M., Gambardella, L.M., : Ant colony system: a cooperative learning approach to the traveling salesman problem. IEEE Transaction on Evolutionary Computation. Vol. 1, No. 1, 1997, 53- 66

[23]. Yimeng yue., Xinwang.,: An improved ant colony optimization algorithm for solving TSP. International Journal of Multimedia and Ubiquitous Engineering vol.10.No.12., 2015, 153-164

[24]. Tang Y.L., Cai Y.G., Yang Q.J.,: Improved ant colony optimization algorithm for solving constraint satisfaction problem. Journal on communications. Vol.36, 5, 2015, 1-6

[25]. Montemanni,R., Gambardella, L.M., Rizzoli, A.E., Donati, A.V.,: Ant colony system for a dynamic vehicle routing problem. Journal of combinatorial optimization.vol 10 ,Issue 4, 2005, 327- 343 